\title{
MS31-01 | Mechanical Properties of Molecular Crystals: The Bigger Picture
}

Naumov, Pance (New York University Abu Dhabi, Abu Dhabi, ARE)

Mechanically reconfigurable molecular crystals-ordered materials that can adapt to variable operating and environmental conditions by deformation, whereby they attain motility or perform work-are quickly shaping up a new research direction in materials science, crystal adaptronics. Properties such as elasticity, superelasticity and ferroelasticity that are normally related to inorganic materials, and phenomena such as shape-memory and selfhealing effects which are well established for soft materials, are increasingly reported for molecular crystals, yet their mechanism, quantification, and relation to the crystal structure in organic crystals are not immediately intelligible to the chemistry and materials science research communities. This lecture will provide a condensed topical overview of the elastic, superelastic and ferroelastic molecular crystals, emerging new classes of materials that bridge the gap between the soft matter and inorganic materials. The occurrence and detection of these unconventional properties, and the underlying structural features of the related molecular materials will be discussed and highlighted together with selected prominent recent examples. 